\title{
Lenguaje e inteligencia de preescolares: Análisis de su relación y factores asociados
}

\author{
LUISA SCHONHAUT B. ${ }^{1}$, MARIANGELA MAGGIOLO L. ${ }^{2}$, MARÍA ELENA HERRERA G. ${ }^{1}$, \\ KERYMA ACEVEDO G. ${ }^{3}$, MÓNICA GARCÍA E. ${ }^{2}$ \\ 1. Pediatra. Departamento Pediatría y Cirugía Infantil Campus Norte, Facultad de Medicina, Universidad de Chile. \\ 2. Fonoaudióloga. Escuela de Fonoaudiología, Facultad de Medicina, Universidad de Chile. \\ 3. Neuropediatra. Departamento Pediatría y Cirugía Infantil Campus Norte, Facultad de Medicina, Universidad de Chile.
}

\begin{abstract}
Preschoolers language and intelligence: Associated factors and relationship analysis

Background: Language and intelligence are brain superior functions and their development is vital for school and future work performance. Objective: To establish the correlation between language and intelligence, in order to identify possible factors involved in preschoolers of low socioeconomical status. Methods: Prospective and blind study that performs specific language tests, audiometry, WPSSI and pediatric-neurological examination to all children between 3-5 year-old attending a daycare, belonging to families of low socioeconomical conditions at the Metropolitan Region of Chile, during 2007. Children with major diseases, explaining language difficulties (LD) were excluded. Results: 61 preschoolers, in which 22 $(36 \%)$ LD were identified. Cognitive performance presented a normal distribution (mean 92,69+15). There was a significant correlation between intelligence and language ( $p$ 0.002). Children with LD had lower cognitive performance, both in verbal ( $p$ 0.004) and in manual tests ( $p$ 0.0007). The family history of LD or learning difficulties and history of developmental delay were associated with a lower language performance ( 0,05 and $p<0.0000$, respectively). Children of mothers with superior studies showed better scores in their cognitive potential ( $\mathrm{p}$ 0.0079). Conclusion: Language and intelligence are significantly associated. Biological and hereditary factors were correlated with LD, whereas social factors (ex: maternal education) were associated with cognitive performance. These findings should be considered in the planification of health politics related with children development, implementing multidisciplinary strategies.

(Key words: language difficulties, intelligence, children development).

Rev Chil Pediatr 2008; 79 (6): 600-606
\end{abstract}

\section{RESUMEN}

Introducción: El lenguaje y la inteligencia son funciones cerebrales superiores cuyo desarrollo es determinante para el desempeño escolar y futuro laboral. Objetivo: Establecer la correlación entre lenguaje e inteligencia, e identificar posibles factores asociados, en preescolares de nivel socio económico bajo.

Trabajo recibido el 27 de agosto de 2008, devuelto para corregir el 28 de octubre de 2008, segunda versión el 29 de noviembre de 2008, aceptado para publicación el 03 de diciembre de 2008.

Proyecto financiado por Concurso de Proyectos de la Sociedad Chilena de Pediatría.

Correspondencia a:

Luisa Schonhaut B.

E-mail: Lschonhaut@med.uchile.cl 
Metodología: Durante el año 2007 en forma prospectiva, simultánea y ciega, se aplicaron pruebas específicas de lenguaje, audiometría, WPSSI y evaluación pediátrica y neurológica completa, a todos los niños de 3 a 5 años, asistentes a un establecimiento de educación preescolar, de nivel socioeconómico bajo, de la Región Metropolitana de Chile. Se excluyeron los niños portadores de patología de base que pudiera dar cuenta de las dificultades de lenguaje (DL). Resultados: La muestra fue conformada por 61 preescolares. En $22(36 \%)$ se identificó DL. El rendimiento cognitivo siguió una distribución normal (promedio 92,69+15). Hubo correlación significativa entre inteligencia y lenguaje ( $\mathrm{p}$ 0,002). Los niños con DL presentaron menor rendimiento cognitivo, tanto verbal (p 0,004) como de ejecución ( $\mathrm{p} 0,0007)$. El antecedente familiar de DL o trastornos de aprendizaje e historia de déficit del DSM se asociaron a menor rendimiento de lenguaje ( $\mathrm{p} 0,05$ y $\mathrm{p}<0,0000)$. Los hijos de madres con educación superior mostraron mejor rendimiento en su potencial cognitivo ( p 0,0079). Conclusión: El lenguaje y la inteligencia se asociaron significativamente; factores hereditarios y biológicos se correlacionaron con DL, mientras que factores sociales con el rendimiento cognitivo. Estos hallazgos debieran considerarse en las políticas de desarrollo infantil, implementando estrategias multisectoriales y multidisciplinarias.

(Palabras clave: Déficit lenguaje, inteligencia, desarrollo infantil).

Rev Chil Pediatr 2008; 79 (6): 600-606

\section{Introducción}

El desarrollo psicomotor (DSM) es la manifestación externa y visible de la maduración del Sistema Nervioso, expresado a través de la adquisición de habilidades cada vez más complejas a lo largo de los primeros años de vida. Si bien el potencial de desarrollo es determinado genéticamente, variables ambientales modulan cuánto de éste va a ser expresado, siendo las funciones cerebrales superiores, como el lenguaje y la cognición, las áreas más vulnerables.

Las dificultades de lenguaje (DL) son aquellos déficits que comprometen los procesos de comprensión y expresión verbal y que interfieren en la interacción comunicativa del niño con su entorno. El diagnóstico definitivo debe ser realizado por un especialista ${ }^{1}$ luego de descartar enfermedades de base, que puedan dar cuenta de la etiología del déficit. Cuando no se encuentra un antecedente etiológico claro, se plantea un Trastorno Específico del Lenguaje, síndrome que corresponde a un espectro heterogéneo de dificultades de distinto grado y características ${ }^{2}$.

Las DL suelen asociarse a dificultades de aprendizaje y trastornos del comportamiento, problemas que en ausencia de una adecuada intervención, pueden persistir hasta la vida adul$\mathrm{ta}^{3-5}$. Artículos recientes se refieren a las comorbilidades asociadas a los trastornos del lenguaje, que afectan el rendimiento global, praxias y/o habilidades motoras; además se describe la presencia de signos neurológicos blandos ${ }^{6}$, e incluso dificultades en las áreas emocional y social ${ }^{7-9}$.

En un estudio reciente se reportó que prácticamente la mitad de los párvulos asistentes a educación preescolar presentaban DL de diversa severidad ${ }^{10}$. Debido a lo expuesto, se formuló un estudio piloto, cuyo objetivo es establecer la asociación del desarrollo de lenguaje con el rendimiento cognitivo, e identificar posibles factores asociados, en una muestra de niños asistentes a un establecimiento de educación parvularia, de estrato socio económico bajo, de la Región Metropolitana de Chile.

\section{Pacientes y Métodos}

Estudio transversal analítico. Durante el año 2007 se evaluó en forma individual, simultánea y ciega, por diferentes especialistas la totalidad de preescolares de 3 a 5 años que asisten regularmente a un establecimiento de educación preescolar, de una comuna urbana del área Norte de la Región Metropolitana de Chile, correspondiendo a 65 niños.

Se dispuso de una sala con las condiciones adecuadas para estas evaluaciones, sin distractores visuales ni auditivos. Se solicitó el consentimiento informado a los padres, que en su totalidad accedió a participar del estudio. 
1. Fonoaudiólogos efectuaron la evaluación del lenguaje aplicando dos pruebas específicas:

- Test de Procesos de Simplificación Fonológica, TEPROSIF ${ }^{11}$, que evalúa el nivel fonológico del lenguaje entre los 3 y los 5 años 11 meses.

- Test Exploratorio de Gramática Española de A. Toronto ${ }^{12}$, que evalúa el desempeño gramatical básico. Este test consta de dos sub-pruebas. Una mide la comprensión y la otra la expresión de determinados aspectos morfosintácticos. Se aplica en el rango de edad de 3 años a 6 años 11 meses.

El desempeño se consideró deficitario si al menos en una de las pruebas o sub-pruebas aplicadas, el rendimiento fue igual o inferior al p10 ó 2 DS bajo la media de su rango etario, dependiendo de cada test.

Para obtener el rendimiento de lenguaje, se sumó el puntaje obtenido en cada una de las pruebas, considerando separadamente las dos sub-pruebas del Test Exploratorio de Gramática Española. Se asignó un puntaje de 1 a 4 puntos por prueba, de modo que 1 punto corresponde a p10 ó -2 DS; 2 a p25 ó -1 DS; 3 a p5075 o promedio y 4 a p90 ó 2 DS.

2. Se efectuó tamizado auditivo modifica$\mathrm{do}^{13}$, con audiómetro MAICO MA 21, en una sala acondicionada para este fin. Se consideró hipoacusia severa cuando el promedio de tonos puros estaba entre 70 y $90 \mathrm{db}$ y profundas sobre este último valor.

3. Psicólogos aplicaron el test WPPSI (Wechsler Preschool and Primary Scale of Intelligence $)^{14}$ para establecer desempeño cognitivo de los niños. Se consideró retraso cognitivo cuando el coeficiente intelectual era inferior a -3 DS de la prueba de origen.

4. Se realizó una evaluación clínica pediátrica y neurológica completa, por pediatras y neurólogos infantiles.

5. Se aplicó una encuesta sociodemográfica, donde se consignaron datos relacionados con el sexo, edad y escolaridad de los padres, antecedentes de pesquisa de rezago del desarrollo psicomotor en los controles de salud previos, edad de ingreso a educación preescolar, ingreso familiar promedio, antecedentes familiares de DL o de aprendizaje. Esta encuesta fue aplicada al cuidador principal del niño.
A través de una entrevista se indagó por la presencia de factores de riesgo sociales, considerando como tal, la escolaridad de los padres igual o inferior a 8 años, extrema pobreza, depresión materna, fallecimiento de alguno de los progenitores, disfunción familiar como violencia intrafamiliar, negligencia y alcoholismo o consumo de drogas en el núcleo familiar.

Una vez completadas las evaluaciones, los resultados individuales fueron discutidos por el equipo multidisciplinario. Se envió un informe personalizado y confidencial a los padres, con la conclusión e intervención sugerida.

Se excluyeron del análisis de este estudio aquellos niños en que se detectó una patología que pudiera dar cuenta del DL, como hipoacusia severa o profunda, retraso cognitivo, retraso generalizado del desarrollo y la presencia de una enfermedad neurológica o congénita conocida.

Se realizaron estadísticas descriptivas y analíticas, de acuerdo a la naturaleza de las variables (test de chi cuadrado, t de student, pruebas de normalidad, etc) Se utilizó el programa estadístico computacional Stata versión 10. El nivel de significación utilizado en las diferentes pruebas fue de $p<0,05$.

\section{Resultados}

Se evaluó la totalidad de los preescolares de entre 3 y 5 años, asistentes al establecimiento de educación preescolar en estudio, correspondiendo a 65 niños. Las características socio demográficas se muestran en la tabla 1.

Se excluyeron 4 niños por presentar una patología de base: tres de ellos en estudio en el nivel terciario. El cuarto niño fue derivado a especialista por retraso cognitivo. No se encontraron niños con hipoacusia severa.

De los 61 preescolares restantes, a 58 (95\%) se les efectuó la totalidad de las pruebas. En $22 / 61$ preescolares $(36 \%)$ se identificó DL sin una causa evidente.

El desempeño cognitivo siguió una curva de distribución normal, con un promedio de 92,69 \pm 15 puntos, lo que corresponde a una significativa desviación a izquierda respecto a valores poblacionales estándares ( $\mathrm{p}<0,0002)$ (figura 1). 
Tabla 1. Caracterización biodemográfica de la población en estudio

\begin{tabular}{|c|c|}
\hline Sexo & $\begin{array}{l}32 \text { masculino } \\
29 \text { femenino }\end{array}$ \\
\hline Edad & $\begin{array}{l}\text { Promedio: } 3 \text { años } 11 \text { meses ( } 3 \text { años } 4 \\
\text { meses a } 5 \text { años } 8 \text { meses) } \\
\text { Mediana: } 3 \text { años } 11 \text { meses }\end{array}$ \\
\hline $\begin{array}{l}\text { Edad ingreso } \\
\text { a educación } \\
\text { preescolar }\end{array}$ & $\begin{array}{l}\text { Promedio: } 2 \text { años } 1 \text { mes (rango } 2 \text { a } 48 \\
\text { meses) } \\
\text { Mediana: } 2 \text { años }\end{array}$ \\
\hline Ingreso familiar & $\begin{array}{l}\text { Promedio: } 243000 \text { (rango } 60000 \text { a } \\
\text { 600.000) } \\
\text { Mediana: } 200000\end{array}$ \\
\hline $\begin{array}{l}\text { Años educación } \\
\text { materna }\end{array}$ & $\begin{array}{l}\text { Promedio: } 11 \text { años (rango : } 0 \text { a } 16 \text { años) } \\
\text { Mediana : } 12 \text { años }\end{array}$ \\
\hline Mamá edad & $\begin{array}{l}\text { Promedio } 27,13 \pm 7,4 \text { (rango: } 15 \text { a } 43 \text { ) } \\
9 \text { madres adolescentes al momento del } \\
\text { nacimiento del hijo }\end{array}$ \\
\hline
\end{tabular}

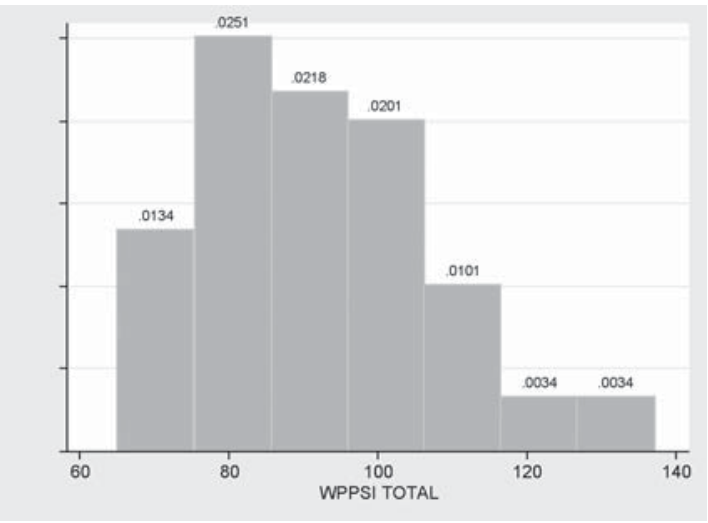

Figura 1. Distribución del coeficiente intelectual de los niños evaluados.

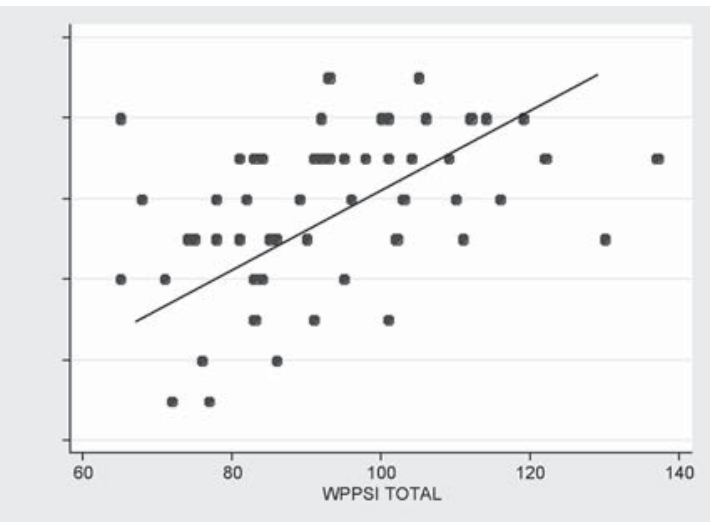

Figura 2. Desempeño en la evaluación de lenguaje versus coeficiente intelectual. $\mathrm{r} 0,5$ p 0,002
Se demostró una correlación entre el rendimiento total del WPPSI y el rendimiento en la evaluación de lenguaje (figura 2) (p 0,002). Los niños con DL presentaron un coeficiente intelectual inferior ( $p 0,0004)$, tanto en las áreas verbal (p 0,004) como de ejecución (p 0,0007) (figuras 3, 4 y 5)

En 9/61 (14,75\%) se pesquisó déficit auditivo, 6 leves y 3 moderados, sólo en un caso la hipoacusia leve fue bilateral, pero no se demostró asociación con DL (p 0,928).

En la tabla 2 se muestra el rendimiento, tanto en la prueba de WPPSI como en la evaluación de lenguaje, en relación a los factores que tuvieron algún grado de significancia estadística. No se encontró correlación con otros

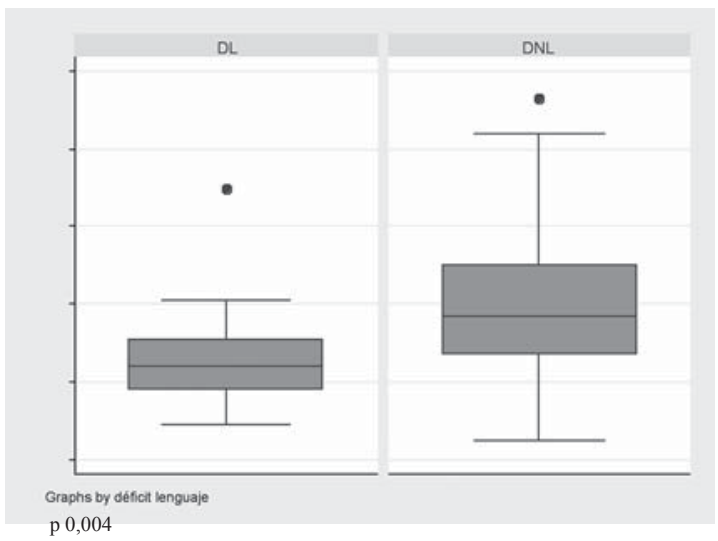

Figura 3. Relación de las DL y el coeficiente intelectual verbal.

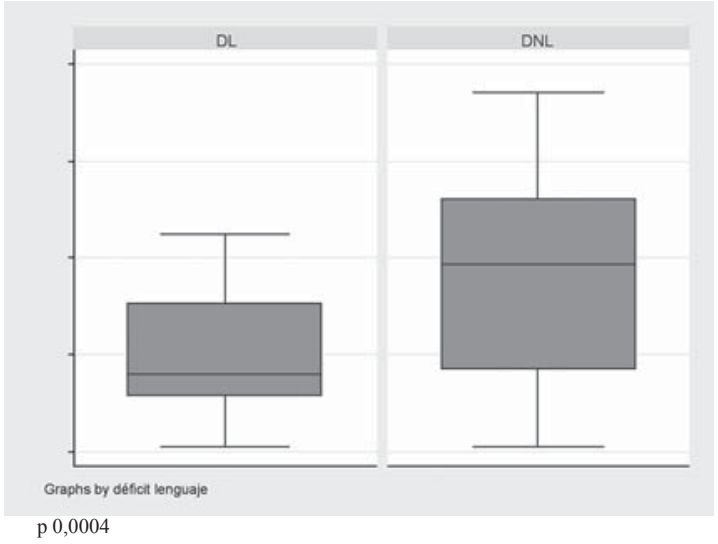

Figura 4. Relación de las DL y el coeficiente intelectual de ejecución. 
Tabla 2. Factores asociados al rendimiento de lenguaje y coeficiente intelectual

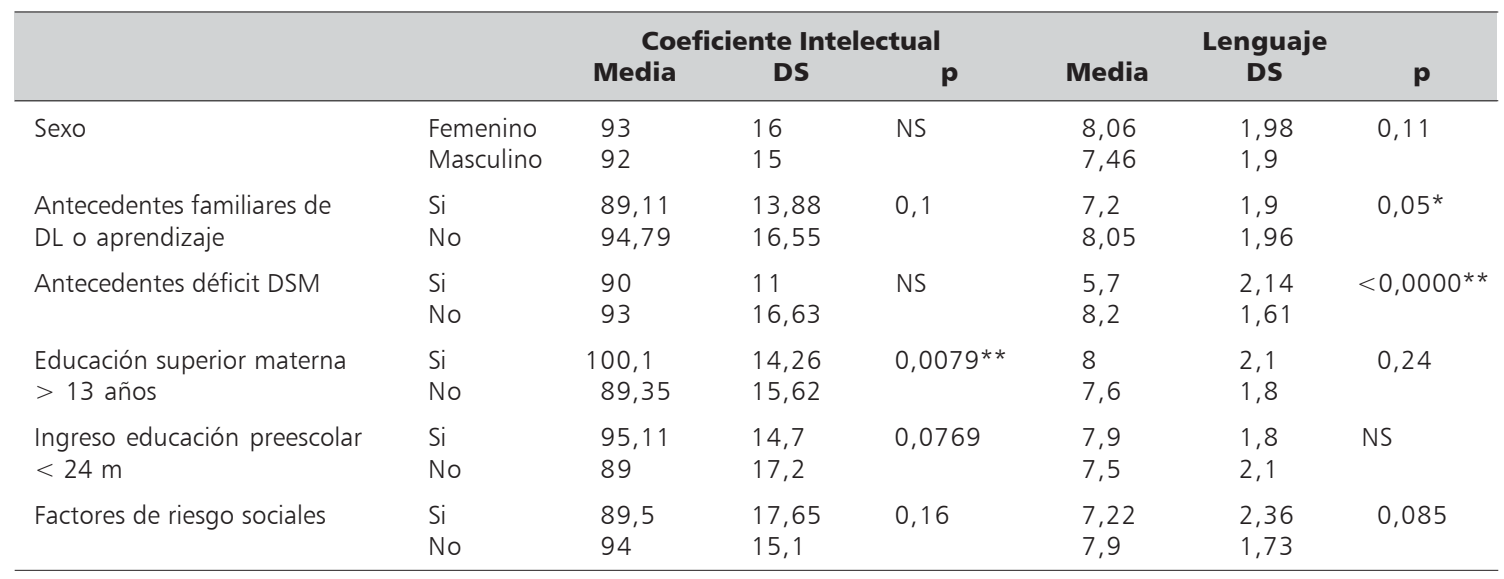

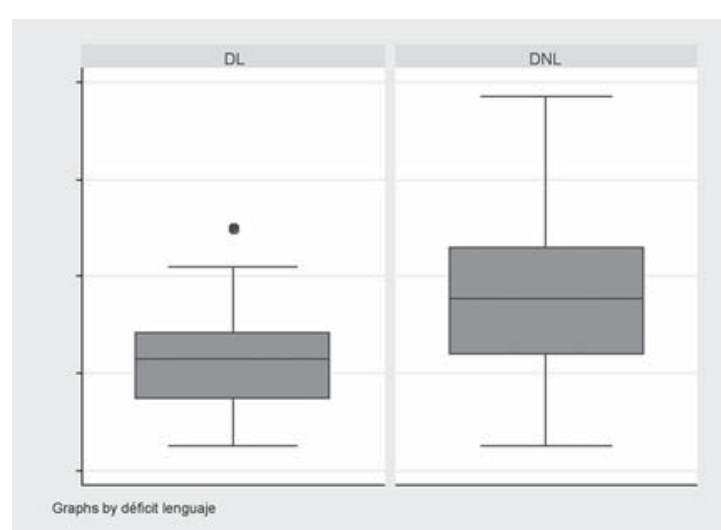

Figura 5. Relación de las DL y el coeficiente intelectual total.

factores estudiados como la escolaridad del padre, edad materna, ingreso familiar promedio, presencia paterna. No hubo diferencia en la edad de ingreso a educación preescolar en relación a la escolaridad materna. Las madres con educación superior tenían menos factores de riesgo sociales en su entorno (p 0,047).

\section{Discusión}

En el presente estudio destaca la alta frecuencia de DL identificados, que alcanza a más de un tercio de la muestra analizada. Esta tasa sobrepasa la prevalencia internacional publica$\mathrm{da}^{15-17}$, sin embargo, es coincidente con el re- porte de la Encuesta Nacional de Salud ${ }^{18}$, y con estudios previos realizados en la misma población ${ }^{10}$, teniendo en cuenta que se excluyeron los niños con trastornos asociados de lenguaje, como enfermedad neurológica o genética.

Al comparar el rendimiento en el WPPSI con los estándares internacionales, llama la atención una desviación hacia puntajes inferiores, aspecto que ha sido evidenciado en sectores de pobreza de la región de las Américas ${ }^{19,20}$.

Se encontró una asociación significativa entre el lenguaje y la inteligencia de los niños. Los niños con DL tenían un rendimiento significativamente inferior en las pruebas de inteligencia tanto verbal como de ejecución. Cada vez existe mayor evidencia respecto a la presencia de comorbilidad en otras áreas del desarrollo en los niños con DL de naturaleza más específi$\mathrm{ca}^{6,21}$, probablemente debido a una inmadurez del sistema nervioso que subyace al desarrollo de distintas áreas incluidas el lenguaje, cognición y motricidad, lo que resalta la importancia de una intervención multidisciplinaria.

Se estudió la asociación de factores sociodemográficos y antecedentes familiares y personales con el desarrollo del lenguaje y la inteligencia. En relación al lenguaje, tuvieron más peso factores biológicos y hereditarios como los antecedentes familiares de DL o de aprendizaje, además de la historia de dificultades del DSM pesquisado en controles de salud, en coincidencia con estudios previos ${ }^{22}$. El antecedente familiar de problemas de lenguaje o de 
aprendizaje, podría ser respaldado por las teorías que estudian una posible base hereditaria del Trastorno Específico del Lenguaje ${ }^{23-25}$.

En cuanto al desarrollo cognitivo, tuvieron más peso factores ambientales y sociales, como la escolaridad materna y, en el límite de la significancia, el ingreso a edades precoces a educación parvularia, hallazgo que confirma la importancia del entorno en el desarrollo infantil. La escolaridad materna mayor o igual a 13 años se asoció además a menor presencia de factores de riesgo sociales como violencia intrafamiliar o negligencia.

Para el correcto análisis de los factores asociados al desarrollo del lenguaje e inteligencia, es importante tener en consideración que los niños provenían de un nivel socio económico bajo. La relación entre pobreza y problemas del desarrollo ha sido ampliamente documentada ${ }^{18,26-31}$. En el área de lenguaje, se ha demostrado la relevancia del nivel educacional ${ }^{32,33}$, manejo del lenguaje y los problemas de salud mental de los cuidadores principales ${ }^{34,35}$.

Sin duda sería de gran interés, ampliar el estudio y replicarlo en niños de distintos sectores socioeconómicos, con el fin de confirmar y especificar los hallazgos; no obstante, dado su relevancia, pensamos que éstos deberían ser considerados en la planificación de políticas relacionadas con el desarrollo de los niños, a través de la implementación de estrategias multidisciplinarias e intersectoriales, con especial énfasis en la detección e intervención integral, de los casos de alto riesgo.

\section{Conclusiones}

Se encontró una significativa correlación entre el lenguaje y la inteligencia. Los niños con DL tuvieron un rendimiento significativamente inferior en las pruebas de inteligencia, tanto en las áreas verbal como no verbal, lo que hace planteable la existencia de comorbilidad de las DL con otras áreas del desarrollo. Al analizar la asociación entre distintos factores con el desarrollo del lenguaje y la inteligencia, destaca que factores biológicos y hereditarios tuvieron asociación con el desempeño en el área de lenguaje, mientras que factores ambientales y sociales tuvieron mayor impacto en el desempeño cognitivo.

Estos factores deberían ser considerados en la planificación de políticas relacionadas con el desarrollo de los niños, a través de la implementación de estrategias multidisciplinarias e intersectoriales, con especial énfasis en la detección e intervención integral, de los casos de alto riesgo.

\section{Agradecimientos}

Las autoras agradecen muy especialmente a las directoras y educadoras del Jardín Infantil Jesús de Belén de la Comuna de Recoleta, por su excelente disposición y valiosa colaboración al desarrollo de este proyecto.

\section{Referencias}

1.- Nelson H, Nygren P, Walker M, Panoscha R: Screening for Speech and Language Delay in Preschool Children: systematic evidence review for the U.S. Preventive Services Task Force. Pediatrics 2006; 117: 298-319.

2.- Leonard L: Children with Specific Language Impairment, 1997, Cambridge, MA: MIT Press.

3.- Law J, Boyle J, Harris F, Harkness A, Nye C: Screening for primary speech and language delay: a systematic review of the literature. Int J Lang Commun Disord 1998; 33 (suppl): 21-3.

4.- Young AR, Beitchman JH, Johnson C, et al: Young adult academic outcomes in a longitudinal sample of early identified language impaired children and control children. Journal of Child Psychology and Psychiatry and Allied Disciplines 2002; 43 (5): 635 45.

5.- Felsenfeld S, Broen P, Mcgue M: A 28-Year FollowUp of Adults With a History of Moderate Phonological Disorder: Educational and Occupational Results. J Speech Hear Res 1994 37: 1341-53.

6.- Webster R, Erdos C, Evans K, et al: The Clinical Spectrum of Developmental Language Impairment in School-Aged Children: Language, Cognitive, and Motor Findings. Pediatrics 2006 118: e1541-e1549. http:/ /pediatrics.aappublications.org/cgi/content/abstract/118/ 5/e1541.

7.- Silva P, Williams $S$, Mcgee R: A longitudinal study of children with development language delay at age three: later intelligence, reading and behaviour problems. Dev Med Child Neurol 1987; 29: 630-40.

8.- Billard C: Le depistage des troubles du langage chez l' enfant. Une contribution á la prévention de l'illetrisme. Arch Pédiatr 2001; 8: 86-91.

9.- Menyuk P, Chesnik M, Liebergott JW: Predicting 
reading problems in at risk children. J Speech Hearing Res1991; 34: 893-903.

10.- Schonhaut L, Maggiolo M, De Barbieri Z, Rojas P, Salgado A: Dificultades de lenguaje en preescolares: concordancia entre el test TEPSI y la evaluación fonoaudiológica. Rev Chil Pediatr 2007; 78 (4): 369 75 .

11.- Maggiolo M, Pávez MM: Test para evaluar los procesos de simplificación fonológica. Ediciones Escuela de Fonoaudiología. Facultad de Medicina, Universidad de Chile. Santiago, Chile 2000.

12.- Pávez MM: Test exploratorio de gramática española de A. Toronto. Ediciones Pontificia Universidad Católica de Chile. Santiago de Chile, 2002.

13.- Internacional Standard Organizartion: International Standards for acoustics. Audiometric test methods. Part 1: Basic Pure tone and bone conduction threshold audiometry. Ginebra ISO 1989, ISO 8253-1: 1989.

14.- Wechler D: WPPSI español: Escala de inteligencia para los niveles preescolar y primario. Editorial El Manual Moderno SA. México 1981.

15.- Rescorla L, Hadicke-wiley M, Escarce E: Epidemiological investigation of expressive language delay at age two. First Lang 1993; 13: 5-22.

16.- Silva PA: The prevalence, stability an significance of developmental language delay in preschool children. Dev Med Child Neurol 1980; 22: 768-77.

17.- Law J, Boyle J, Harris F, Harkness A, Nye C: Prevalence and language delay: findings from a sistematic review of the literature. Int J Lang Comm Dis 2000; 33 (suppl): S21-3.

18.- Subsecretaría de Salud Pública División de Planificación Sanitaria: II Encuesta de Calidad de Vida y Salud, Chile 2006. Disponible en: http://epi.minsal.cl/ epi/html/sdesalud/calidaddevida2006Informe\%20Final $\% 20$ Encuesta $\% 20$ de $\% 20$ Calidad $\% 20$ de $\% 20$ Vida $\%$ 20y\%20Salud\%202006.pdf

19.- Lipina S, Martelli M, Vuelta B, et al: Pobreza y desempeño ejecutivo en alumnos preescolares de la Ciudad de Buenos Aires (República Argentina). Interdisciplinaria 2004; 21 (2): 153-93.

20.- Di Iorio S, Urrutia M, Rodrigo M: Desarrollo psicológico, nutrición y pobreza (Argentina). Rev Chil Pediatr 2000; 71 (3): 263-74.

21.- Downey D, Mraz R, Knott M, Knuston C, Holte L, Van Dyke D: Clinical Psychologist Diagnosis and Evaluation of Children Who Are Not Talking. Inf Young Children 2002; 15 (2): 38-48.

22.- Schonhaut L, Rojas P, Kaempffer A: Factores de riesgo asociados a déficit del desarrollo psicomotor en preescolares de nivel socioeconómico bajo. Rev Chil
Pediatr 2005; 76 (6): 589-98.

23.- Toppelberg CO, Shapiro T: Language Disorder: A 10year research update review. J Am Acad Child Adol Psychiatry 2000; 39: 143-52.

24.- SLI Consortium: Highly significant linkage to the SLI1 locus in an expanded sample of individuals affected by specific language impairment. Am J Hum Genet 2004; 74: 1225-38.

25.- Villanueva P, De Barbieri Z, Palomino H, Palomino H: Alta prevalencia de trastorno específico de lenguaje en isla Robinson Crusoe y probable efecto fundador. Rev Méd Chile 2008; 136 (2): 186-92.

26.- Montenegro H, Guajardo $H$ : Psiquiatría del niño y del adolescente. $2^{\circ}$ edición. Editorial Mediterráneo, Santiago, Chile, 2000.

27.- Halpern R, Giugliani E, Victora C, Barros F, Horta B: Fatores de risco para suspeita de atraso no desenvolvimento neuropsicomotor aos 12 meses de vida. Rev Chil Pediatr 2002; 73 (5): 529-39.

28.- Stanton-Chapman T, Chapman D, Bainbridge $N$, Scott $K$ : Identification of early risk factors for language impairment. Research in Developmental Disabilities 2002; 23 (6): 390-405.

29.- Hooper S, Burchinal M, Roberts Je, Zeisel S, Neebe E: Social and family risk factors for infant development at one year: An application of the cumulative risk model. Journal of Applied Developmental Psychology 1998; 19 (1): 85-96.

30.- Michelini M, Rodríguez S, Montiel S, et al: Apoyo sanitario interdisciplinario en educación inicial. Rev Chil Pediatr 2000; 71 (2): 154-76.

31.- Evans $G$, English $K$ : The Environment of Poverty: Multiple Stressor Exposure, Psychophysiological Stress, and Socioemotional Adjustment. Child Development, 2002; 73 (4): 1238-48.

32.- Paul R: Profiles of toddlers with slow expressive language development. Topics in Language Disorders 1991; 11: 1-13.

33.- Tomblin B, Records N, Buckwalter P, Zhang $X$, Smith E, O'brien M: Prevalence of Specific Language Impairment in Kindergarten Children. Journal of speech and Hearing Research; 1997 (40): 1245-60.

34.- Sameroff A, Seifer R, Baldwin \& Baldwin: Stability of Intelligence from Preschool to Adolescence: The Influence of Social and Family Risk Factors. Child Development 1993; 64 (1): 80-97.

35.- Horwitz S, Irwin J, Briggs-gowan M, Bosson $H$, Mendoza J, Carter A: Language delay in a community cohort of young children. Journal of the American Academy of child and adolescent psychiatry 2003; 42 (8): $932-40$ 